ISSN (Print): 2077-9879

ISSN (Online): 2617-2658

\title{
Eurasian
}

Mathematical

Journal

\section{1, Volume 12, Number 1}

Founded in 2010 by

the L.N. Gumilyov Eurasian National University

in cooperation with

the M.V. Lomonosov Moscow State University

the Peoples' Friendship University of Russia (RUDN University)

the University of Padua

Starting with 2018 co-funded

by the L.N. Gumilyov Eurasian National University

and

the Peoples' Friendship University of Russia (RUDN University)

Supported by the ISAAC

(International Society for Analysis, its Applications and Computation) and

by the Kazakhstan Mathematical Society

Published by

the L.N. Gumilyov Eurasian National University

Nur-Sultan, Kazakhstan 


\title{
EURASIAN MATHEMATICAL JOURNAL Editorial Board
}

\author{
Editors-in-Chief \\ V.I. Burenkov, M. Otelbaev, V.A. Sadovnichy \\ Vice-Editors-in-Chief
}

K.N. Ospanov, T.V. Tararykova

Editors

Sh.A. Alimov (Uzbekistan), H. Begehr (Germany), T. Bekjan (China), O.V. Besov (Russia), N.K. Bliev (Kazakhstan), N.A. Bokayev (Kazakhstan), A.A. Borubaev (Kyrgyzstan), G. Bourdaud (France), A. Caetano (Portugal), M. Carro (Spain), A.D.R. Choudary (Pakistan), V.N. Chubarikov (Russia), A.S. Dzumadildaev (Kazakhstan), V.M. Filippov (Russia), H. Ghazaryan (Armenia), M.L. Goldman (Russia), V. Goldshtein (Israel), V. Guliyev (Azerbaijan), D.D. Haroske (Germany), A. Hasanoglu (Turkey), M. Huxley (Great Britain), P. Jain (India), T.Sh. Kalmenov (Kazakhstan), B.E. Kangyzhin (Kazakhstan), K.K. Kenzhibaev (Kazakhstan), S.N. Kharin (Kazakhstan), E. Kissin (Great Britain), V. Kokilashvili (Georgia), V.I. Korzyuk (Belarus), A. Kufner (Czech Republic), L.K. Kussainova (Kazakhstan), P.D. Lamberti (Italy), M. Lanza de Cristoforis (Italy), F. Lanzara (Italy), V.G. Maz'ya (Sweden), K.T. Mynbayev (Kazakhstan), E.D. Nursultanov (Kazakhstan), R. Oinarov (Kazakhstan), I.N. Parasidis (Greece), J. Pečarić (Croatia), S.A. Plaksa (Ukraine), L.E. Persson (Sweden), E.L. Presman (Russia), M.A. Ragusa (Italy), M.D. Ramazanov (Russia), M. Reissig (Germany), M. Ruzhansky (Great Britain), M.A. Sadybekov (Kazakhstan), S. Sagitov (Sweden), T.O. Shaposhnikova (Sweden), A.A. Shkalikov (Russia), V.A. Skvortsov (Poland), G. Sinnamon (Canada), E.S. Smailov (Kazakhstan), V.D. Stepanov (Russia), Ya.T. Sultanaev (Russia), D. Suragan (Kazakhstan), I.A. Taimanov (Russia), J.A. Tussupov (Kazakhstan), U.U. Umirbaev (Kazakhstan), Z.D. Usmanov (Tajikistan), N. Vasilevski (Mexico), Dachun Yang (China), B.T. Zhumagulov (Kazakhstan)

\section{Managing Editor}

A.M. Temirkhanova

(C) The L.N. Gumilyov Eurasian National University 


\section{Aims and Scope}

The Eurasian Mathematical Journal (EMJ) publishes carefully selected original research papers in all areas of mathematics written by mathematicians, principally from Europe and Asia. However papers by mathematicians from other continents are also welcome.

From time to time the EMJ publishes survey papers.

The EMJ publishes 4 issues in a year.

The language of the paper must be English only.

The contents of the EMJ are indexed in Scopus, Web of Science (ESCI), Mathematical Reviews, MathSciNet, Zentralblatt Math (ZMATH), Referativnyi Zhurnal - Matematika, Math-Net.Ru.

The EMJ is included in the list of journals recommended by the Committee for Control of Education and Science (Ministry of Education and Science of the Republic of Kazakhstan) and in the list of journals recommended by the Higher Attestation Commission (Ministry of Education and Science of the Russian Federation).

\section{Information for the Authors}

Submission. Manuscripts should be written in LaTeX and should be submitted electronically in DVI, PostScript or PDF format to the EMJ Editorial Office through the provided web interface (www.enu.kz).

When the paper is accepted, the authors will be asked to send the tex-file of the paper to the Editorial Office.

The author who submitted an article for publication will be considered as a corresponding author. Authors may nominate a member of the Editorial Board whom they consider appropriate for the article. However, assignment to that particular editor is not guaranteed.

Copyright. When the paper is accepted, the copyright is automatically transferred to the EMJ. Manuscripts are accepted for review on the understanding that the same work has not been already published (except in the form of an abstract), that it is not under consideration for publication elsewhere, and that it has been approved by all authors.

Title page. The title page should start with the title of the paper and authors' names (no degrees). It should contain the Keywords (no more than 10), the Subject Classification (AMS Mathematics Subject Classification (2010) with primary (and secondary) subject classification codes), and the Abstract (no more than 150 words with minimal use of mathematical symbols).

Figures. Figures should be prepared in a digital form which is suitable for direct reproduction.

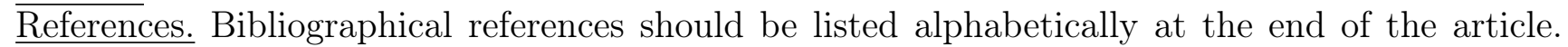
The authors should consult the Mathematical Reviews for the standard abbreviations of journals' names.

Authors' data. The authors' affiliations, addresses and e-mail addresses should be placed after the References.

Proofs. The authors will receive proofs only once. The late return of proofs may result in the paper being published in a later issue.

Offprints. The authors will receive offprints in electronic form. 


\section{Publication Ethics and Publication Malpractice}

For information on Ethics in publishing and Ethical guidelines for journal publication see http://www.elsevier.com/publishingethics and http://www.elsevier.com/journal-authors/ethics.

Submission of an article to the EMJ implies that the work described has not been published previously (except in the form of an abstract or as part of a published lecture or academic thesis or as an electronic preprint, see http:/ /www.elsevier.com/postingpolicy), that it is not under consideration for publication elsewhere, that its publication is approved by all authors and tacitly or explicitly by the responsible authorities where the work was carried out, and that, if accepted, it will not be published elsewhere in the same form, in English or in any other language, including electronically without the written consent of the copyright-holder. In particular, translations into English of papers already published in another language are not accepted.

No other forms of scientific misconduct are allowed, such as plagiarism, falsification, fraudulent data, incorrect interpretation of other works, incorrect citations, etc. The EMJ follows the Code of Conduct of the Committee on Publication Ethics (COPE), and follows the COPE Flowcharts for Resolving Cases of Suspected Misconduct (http://publicationethics.org/files/u2/NewCode.pdf). To verify originality, your article may be checked by the originality detection service CrossCheck http://www.elsevier.com/editors/plagdetect.

The authors are obliged to participate in peer review process and be ready to provide corrections, clarifications, retractions and apologies when needed. All authors of a paper should have significantly contributed to the research.

The reviewers should provide objective judgments and should point out relevant published works which are not yet cited. Reviewed articles should be treated confidentially. The reviewers will be chosen in such a way that there is no conflict of interests with respect to the research, the authors and/or the research funders.

The editors have complete responsibility and authority to reject or accept a paper, and they will only accept a paper when reasonably certain. They will preserve anonymity of reviewers and promote publication of corrections, clarifications, retractions and apologies when needed. The acceptance of a paper automatically implies the copyright transfer to the EMJ.

The Editorial Board of the EMJ will monitor and safeguard publishing ethics. 


\section{The procedure of reviewing a manuscript, established by the Editorial Board of the Eurasian Mathematical Journal}

\section{Reviewing procedure}

1.1. All research papers received by the Eurasian Mathematical Journal (EMJ) are subject to mandatory reviewing.

1.2. The Managing Editor of the journal determines whether a paper fits to the scope of the EMJ and satisfies the rules of writing papers for the EMJ, and directs it for a preliminary review to one of the Editors-in-chief who checks the scientific content of the manuscript and assigns a specialist for reviewing the manuscript.

1.3. Reviewers of manuscripts are selected from highly qualified scientists and specialists of the L.N. Gumilyov Eurasian National University (doctors of sciences, professors), other universities of the Republic of Kazakhstan and foreign countries. An author of a paper cannot be its reviewer.

1.4. Duration of reviewing in each case is determined by the Managing Editor aiming at creating conditions for the most rapid publication of the paper.

1.5. Reviewing is confidential. Information about a reviewer is anonymous to the authors and is available only for the Editorial Board and the Control Committee in the Field of Education and Science of the Ministry of Education and Science of the Republic of Kazakhstan (CCFES). The author has the right to read the text of the review.

1.6. If required, the review is sent to the author by e-mail.

1.7. A positive review is not a sufficient basis for publication of the paper.

1.8. If a reviewer overall approves the paper, but has observations, the review is confidentially sent to the author. A revised version of the paper in which the comments of the reviewer are taken into account is sent to the same reviewer for additional reviewing.

1.9. In the case of a negative review the text of the review is confidentially sent to the author.

1.10. If the author sends a well reasoned response to the comments of the reviewer, the paper should be considered by a commission, consisting of three members of the Editorial Board.

1.11. The final decision on publication of the paper is made by the Editorial Board and is recorded in the minutes of the meeting of the Editorial Board.

1.12. After the paper is accepted for publication by the Editorial Board the Managing Editor informs the author about this and about the date of publication.

1.13. Originals reviews are stored in the Editorial Office for three years from the date of publication and are provided on request of the CCFES.

1.14. No fee for reviewing papers will be charged.

\section{Requirements for the content of a review}

2.1. In the title of a review there should be indicated the author(s) and the title of a paper.

2.2. A review should include a qualified analysis of the material of a paper, objective assessment and reasoned recommendations.

2.3. A review should cover the following topics:

- compliance of the paper with the scope of the EMJ;

- compliance of the title of the paper to its content;

- compliance of the paper to the rules of writing papers for the EMJ (abstract, key words and phrases, bibliography etc.);

- a general description and assessment of the content of the paper (subject, focus, actuality of the topic, importance and actuality of the obtained results, possible applications);

- content of the paper (the originality of the material, survey of previously published studies on the topic of the paper, erroneous statements (if any), controversial issues (if any), and so on); 
- exposition of the paper (clarity, conciseness, completeness of proofs, completeness of bibliographic references, typographical quality of the text);

- possibility of reducing the volume of the paper, without harming the content and understanding of the presented scientific results;

- description of positive aspects of the paper, as well as of drawbacks, recommendations for corrections and complements to the text.

2.4. The final part of the review should contain an overall opinion of a reviewer on the paper and a clear recommendation on whether the paper can be published in the Eurasian Mathematical Journal, should be sent back to the author for revision or cannot be published. 


\section{Web-page}

The web-page of the EMJ is www.emj.enu.kz. One can enter the web-page by typing Eurasian Mathematical Journal in any search engine (Google, Yandex, etc.). The archive of the web-page contains all papers published in the EMJ (free access).

\section{Subscription}

Subscription index of the EMJ 76090 via KAZPOST.

\section{E-mail}

eurasianmj@yandex.kz

The Eurasian Mathematical Journal (EMJ)

The Nur-Sultan Editorial Office

The L.N. Gumilyov Eurasian National University

Building no. 3

Room 306a

Tel.: +7-7172-709500 extension 33312

13 Kazhymukan St

010008 Nur-Sultan, Kazakhstan

The Moscow Editorial Office

The Peoples' Friendship University of Russia

(RUDN University)

Room 562

Tel.: +7-495-9550968

3 Ordzonikidze St

117198 Moscow, Russia 


\title{
SHAVKAT ARIFJANOVICH ALIMOV
}

\author{
(to the 75 th birthday)
}

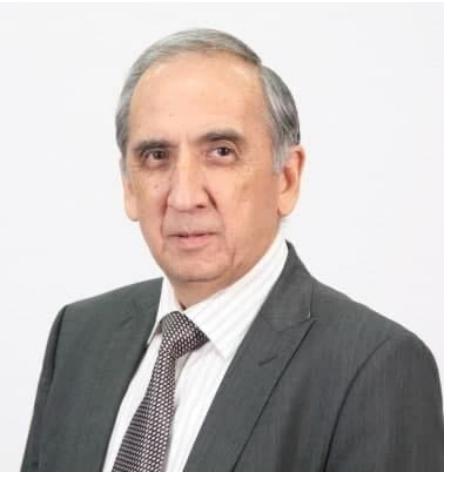

Shavkat Arifjanovich Alimov was born on March 2, 1945 in the city of Nukus, Uzbekistan. In 1968, he graduated from the Department of Mathematics of Physical Faculty of the M.V. Lomonosov Moscow State University (MSU), receiving a diploma with honors. From 1968 to 1970, he was a post-graduate student in the same department under the supervision of Professor V.A. Il'in. He defended his PhD thesis in 1970. In May 1973, at the age of 28 , he defended his doctoral thesis devoted to equations of mathematical physics. In 1973, for research on the spectral theory, he was awarded the highest youth prize of the USSR.

From 1974 to 1984, he worked as a professor in the Department of General Mathematics at the Faculty of Computational Mathematics and Cybernetics. In 1984, Sh.A. Alimov joined the Tashkent State University (TSU) as a professor. From 1985 to 1987 he worked as the Rector of the Samarkand State University, from 1987 to 1990 - the Rector of the TSU, from 1990 to 1992 - the Minister of Higher and Secondary Special Education of the Republic of Uzbekistan. From 1992 to 1994, he headed the Department of Mathematical Physics of the TSU.

After some years of diplomatic work, he continued his academic career as a professor of the Department of Mathematical Physics at the National University of Uzbekistan (NUU). From the first days of the opening of the Tashkent branch of the MSU in 2006, he worked as a professor in the Department of Applied Mathematics. From 2012 to 2017, he headed the Laboratory of Mathematical Modeling of the Malaysian Institute of Microelectronic Systems in Kuala Lumpur. From 2017 to 2019, he worked as a professor at the Department of Differential Equations and Mathematical Physics of the NUU. From 2019 to the present, Sh.A. Alimov is a Scientific Consultant at the Center for Intelligent Software Systems, and an adviser to the Rector of the NUU.

The main scientific activity of Sh.A. Alimov is connected with the spectral theory of partial differential equations and the theory of boundary value problems for equations of mathematical physics. He obtained series of remarkable results in these fields. They cover many important problems of the theory of Schrodinger equations with singular potentials, the theory of boundary control of the heat transfer process, the mathematical problems of peridynamics related to the theory of hypersingular integrals.

In 1984, Sh.A. Alimov was elected a corresponding member and in 2000 an academician of the Academy of Sciences of Uzbekistan. He was awarded several prestigious state prizes.

Sh.A. Alimov has over 150 published scientific and a large number of educational works. Among his pupils there are 10 doctors of sciences and more than 20 candidates of sciences (PhD) working at universities of Uzbekistan, Russia, USA, Finland, and Malaysia.

For about thirty years, Sh.A. Alimov has been actively involved in the reform of mathematical school education.

Sh.A. Alimov meets his 75th birthday in the prime of his life, and the Editorial Board of the Eurasian Mathematical Journal heartily congratulates him on his jubilee and wishes him good health, new successes in scientific and pedagogical activity, family well-being and long years of fruitful life. 


\title{
Short communications
}

\author{
EURASIAN MATHEMATICAL JOURNAL
}

ISSN 2077-9879

Volume 12, Number 1 (2021), 92 - 96

\section{ON INTEGRAL OPERATORS WITH HOMOGENEOUS KERNELS IN MORREY SPACES}

\author{
O.G. Avsyankin
}

Communicated by E.D. Nursultanov

Key words: Morrey space, integral operator, homogeneous kernel, boundedness, symbol, invertibility.

\section{AMS Mathematics Subject Classification: 47G10.}

\begin{abstract}
We consider integral operators with homogeneous kernels in Morrey spaces. For such operators we obtain sufficient conditions of their boundedness. Moreover, for an operator, which is the sum of the identity operator and an operator with a homogeneous kernel, we prove the invertibility criterion.
\end{abstract}

DOI: https://doi.org/10.32523/2077-9879-2021-12-1-92-96

\section{Introduction}

At the present time, there are quite a few papers dealing with integral operators with homogeneous kernels (e.g., see [1]-[3], [5], [11], [12] and the bibliography therein). For such operators, criteria of invertibility and Fredholm property were obtained, Banach algebra generated by these operators were studied, the necessary and sufficient conditions for the projection method to apply were found and spectral characteristics were described. However, all of the above results relate to operators acting in $L_{p}$-spaces. In other spaces the operators with homogeneous kernels were considered only in some papers. For example, in [14] operators with homogeneous kernels were studied in grand Lebesgue spaces.

The present paper is devoted to integral operators with homogeneous kernels in Morrey spaces. The study of Morrey spaces and operators in these spaces goes back to [13]. During the last three decades there was a lot of activity in this area (e. g., see [6], [7] and the bibliography therein). At the same time, much attention was paid to operators of classical analysis, such as maximal operator, Riesz potential, singular integral operator, Hardy operator. In recent years, there is a tendency to study convolution operators (see [8], [9], [4]).

In this paper we consider an integral operator $K$ with a homogeneous kernel of degree -1 . For such an operator we establish sufficient conditions for the boundedness in Morrey spaces on the half-axis. Moreover, for the operator $I+K$ we construct a symbol and in terms of this symbol we obtain the invertibility criterion of this operator. 


\section{Preliminaries}

Let $\mathbb{R}_{+}=(0, \infty), 1 \leqslant p \leqslant \infty$, and $D \subseteq \mathbb{R}_{+}$be a measurable set. Then $L_{p}(D)$ is the space (of classes) of measurable complex-valued functions with the norm

$$
\|f\|_{L_{p}(D)}=\left(\int_{D}|f(x)|^{p} d x\right)^{1 / p}, 1 \leqslant p<\infty ; \quad\|f\|_{L_{\infty}(D)}=\underset{x \in D}{\operatorname{essipup}}|f(x)| .
$$

A function $f \in L_{p}^{\text {loc }}\left(\mathbb{R}_{+}\right)$if $f \in L_{p}(K)$ for any compact $K \subset \mathbb{R}_{+}$. For any $x, r \in \mathbb{R}_{+}$put $J(x, r)=$ $(x-r, x+r) \cap \mathbb{R}_{+}$.

Definition 1. Let $1 \leqslant p \leqslant \infty$ and $\lambda \in \mathbb{R}$. It is said that a function $f$ belongs to $L_{p, \lambda}\left(\mathbb{R}_{+}\right)$if $f \in L_{p}^{l o c}\left(\mathbb{R}_{+}\right)$and

$$
\|f\|_{L_{p, \lambda}\left(\mathbb{R}_{+}\right)} \equiv\|f\|_{p, \lambda}=\sup _{x, r \in \mathbb{R}_{+}} \frac{\|f\|_{L_{p}(J(x, r))}}{r^{\lambda}}<\infty .
$$

With respect to the usual linear operations and norm (2.1) the set $L_{p, \lambda}\left(\mathbb{R}_{+}\right)$forms a Banach space, which is called a Morrey space.

The spaces $L_{p, \lambda}\left(\mathbb{R}_{+}\right)$are nontrivial, i. e. they consist not only of functions equivalent to zero on $\mathbb{R}_{+}$if and only if $0 \leqslant \lambda \leqslant 1 / p$. For $\lambda=0$ and $\lambda=1 / p$ Morrey spaces coincide with $L_{p}$-spaces:

$$
L_{p, 0}\left(\mathbb{R}_{+}\right)=L_{p}\left(\mathbb{R}_{+}\right), \quad L_{p, \frac{1}{p}}\left(\mathbb{R}_{+}\right)=L_{\infty}\left(\mathbb{R}_{+}\right) .
$$

Since the integral operators with homogeneous kernels in $L_{p}$-spaces are well studied, taking into account equalities (2.2), we will not consider the cases $\lambda=0$ и $\lambda=1 / p$.

Denote by $\mathcal{L}\left(L_{p, \lambda}\left(\mathbb{R}_{+}\right)\right)$the set of all bounded linear operators acting in $L_{p, \lambda}\left(\mathbb{R}_{+}\right)$. Since $L_{p, \lambda}\left(\mathbb{R}_{+}\right)$ is a Banach space, the set $\mathcal{L}\left(L_{p, \lambda}\left(\mathbb{R}_{+}\right)\right)$with the usual operations of addition and multiplication and with the usual operator norm forms a Banach algebra.

\section{Main results}

In the space $L_{p, \lambda}\left(\mathbb{R}_{+}\right)$we consider the operator

$$
(K \varphi)(x)=\int_{0}^{\infty} k(x, y) \varphi(y) d y, \quad x \in \mathbb{R}_{+}
$$

where the function $k(x, y)$ is defined on $\mathbb{R}_{+} \times \mathbb{R}_{+}$and satisfies the following conditions:

$1^{\circ}$ homogeneity of degree -1 , i. e.

$$
k(\alpha x, \alpha y)=\alpha^{-1} k(x, y), \quad \forall \alpha>0 ;
$$

$2^{\circ}$ integrability, i. e.

$$
\kappa:=\int_{0}^{\infty}|k(1, y)| y^{-1 / p+\lambda} d y<\infty
$$

Theorem 3.1. Let $1 \leqslant p<\infty, 0<\lambda<1 / p$, and a function $k$ satisfy conditions $1^{\circ}$ and $2^{\circ}$. Then the operator $K$ is bounded in the space $L_{p, \lambda}\left(\mathbb{R}_{+}\right)$and the inequality

$$
\|K \varphi\|_{p, \lambda} \leqslant \kappa\|\varphi\|_{p, \lambda}
$$

holds for every function $\varphi \in L_{p, \lambda}\left(\mathbb{R}_{+}\right)$. 
Let $1 \leqslant p<\infty, 0<\lambda<1 / p$. Denote by $\mathfrak{A}_{0}$ the set consisting of all operators $c I+K$, where $c \in \mathbb{C}, I$ is an identity operator, $K$ is an operator of form (3.1). Let $K_{1}$ and $K_{2}$ be two operators with kernels $k_{1}(x, y)$ and $k_{2}(x, y)$ respectively. Their composition $K=K_{1} K_{2}$ is an integral operator of the same form with kernel

$$
k(x, y)=\int_{0}^{\infty} k_{1}(x, t) k_{2}(t, y) d t .
$$

It is easy to see that the function $k(x, y)$ satisfies conditions $1^{\circ}$ and $2^{\circ}$. Therefore the operation of multiplication is closed on the set $\mathfrak{A}_{0}$. It is easy to verify that the set $\mathfrak{A}_{0}$ equipped with usual operations of addition, multiplication, multiplication of operators by complex numbers and the following norm

$$
\|c I+K\|_{\mathfrak{A}_{0}}=|c|+\int_{0}^{\infty}|k(1, y)| y^{-1 / p+\lambda} d y,
$$

is a Banach algebra. This algebra is commutative because for any functions $k_{1}(x, y)$ and $k_{2}(x, y)$, which satisfy condition $1^{\circ}$, the equality

$$
\int_{0}^{\infty} k_{1}(x, t) k_{2}(t, y) d t=\int_{0}^{\infty} k_{2}(x, t) k_{1}(t, y) d t
$$

holds (see, e.g., [12, p. 381]).

Let us consider the function

$$
\sigma_{c I+K}(\xi)=c+\int_{0}^{\infty} k(1, y) y^{-1 / p+\lambda+i \xi} d y, \quad \xi \in \mathbb{R} .
$$

We call this function the symbol of the operator $c I+K$. The symbol $\sigma_{c I+K}(\xi)$ can be written in another form. Put

$$
h(t)=k\left(1, e^{t}\right) e^{t / p^{\prime}+t \lambda} .
$$

From condition $2^{\circ}$ it follows that $h \in L_{1}(\mathbb{R})$. It is easy to establish that

$$
\sigma_{c I+K}(\xi)=c+\widehat{h}(\xi)
$$

where $\widehat{h}(\xi)=\int_{-\infty}^{\infty} h(t) e^{i \xi t} d t$ is the Fourier transform of the function $h$.

Further we shall denote by $\mathcal{W}(\mathbb{R})$ the Wiener algebra, i. e. the set of all functions of the form $c+\widehat{f}(\xi)$, where $c \in \mathbb{C}$ and $f \in L_{1}(\mathbb{R})$. It is known that with pointwise algebraic operations and the norm

$$
\|c+\widehat{f}\|_{\mathcal{W}}=|c|+\|f\|_{1}
$$

the set $\mathcal{W}(\mathbb{R})$ is a commutative Banach algebra. Taking into account formulas (3.2)-(3.4), we get the following proposition.

Lemma 3.1. The mapping

$$
\mathcal{S}: \mathfrak{A}_{0} \rightarrow \mathcal{W}(\mathbb{R}), \quad c I+K \rightarrow \sigma_{c I+K}(\xi)
$$

is an isometric isomorphism. 
Denote by $\mathcal{M}\left(\mathfrak{A}_{0}\right)$ and $\mathcal{M}(\mathcal{W}(\mathbb{R}))$ the spaces of maximal ideals of Banach algebras $\mathfrak{A}_{0}$ and $\mathcal{W}(\mathbb{R})$ respectively. Since the algebra $\mathfrak{A}_{0}$ is isometrically isomorphic to the algebra $\mathcal{W}(\mathbb{R})$ then the space $\mathcal{M}\left(\mathfrak{A}_{0}\right)$ is homeomorphic to the space $\mathcal{M}(\mathcal{W}(\mathbb{R}))$. Denote by $\dot{\mathbb{R}}$ the one-point compactification of the locally compact space $\mathbb{R}$. It is known $([10$, p. 20]) that the space $\mathcal{M}(\mathcal{W}(\mathbb{R}))$ is homeomorphic to the compact $\dot{\mathbb{R}}$. Therefore the space $\mathcal{M}\left(\mathfrak{A}_{0}\right)$ is also homeomorphic to the compact $\dot{\mathbb{R}}$. Under this homeomorphism each point $\xi_{0} \in \mathbb{R}$ corresponds to the ideal $\mathcal{M}_{\xi_{0}} \in \mathcal{M}\left(\mathfrak{A}_{0}\right)$ consisting of all operators $c I+K$ such that $\sigma_{c I+K}\left(\xi_{0}\right)=0$. As a result, we obtain the invertibility criterion of the operator $c I+K$ in the algebra $\mathfrak{A}_{0}$.

Lemma 3.2. The operator $c I+K$ is invertible in the algebra $\mathfrak{A}_{0}$ if and only if

$$
\sigma_{c I+K}(\xi) \neq 0, \quad \forall \xi \in \dot{\mathbb{R}}
$$

Lemma 3.2 implies that condition (3.5) is sufficient for invertibility of the operator $c I+K$ in $\mathcal{L}\left(L_{p, \lambda}\left(\mathbb{R}_{+}\right)\right)$. It is much more difficult to obtain the necessary condition, because if the operator $c I+K$ is invertible in $\mathcal{L}\left(L_{p, \lambda}\left(\mathbb{R}_{+}\right)\right)$this does not mean that $(c I+K)^{-1} \in \mathfrak{A}_{0}$.

Denote by $\mathfrak{A}$ the least closed subalgebra of the Banach algebra $\mathcal{L}\left(L_{p, \lambda}\left(\mathbb{R}_{+}\right)\right)$containing all operators $c I+K$, where $c \in \mathbb{C}$ and $K$ is an operator of form (3.1). This algebra is the closure of the set $\mathfrak{A}_{0}$ in the uniform operator topology. Note that the algebra $\mathfrak{A}$ is commutative.

Lemma 3.3. Let $K$ be an operator of form (3.1). If the operator $c I+K$ is invertible in $\mathcal{L}\left(L_{p, \lambda}\left(\mathbb{R}_{+}\right)\right)$ then the operator $(c I+K)^{-1}$ belongs to the algebra $\mathfrak{A}$.

Taking into account that the algebra $\mathfrak{A}_{0}$ is densely embedded in the algebra $\mathfrak{A}$, we get the following lemma.

Lemma 3.4. Let $K$ be an operator of form (3.1). If the operator $c I+K$ is invertible in $\mathfrak{A}$ then the operator $(c I+K)^{-1}$ belongs to the algebra $\mathfrak{A}_{0}$.

Lemma 3.2, Lemma 3.3 and Lemma 3.4 imply the following main result.

Theorem 3.2. Let $K$ be an operator of form (3.1). The operator $c I+K$ is invertible in $\mathcal{L}\left(L_{p, \lambda}\left(\mathbb{R}_{+}\right)\right)$ if and only if condition (3.5) holds.

\section{Acknowledgments}

The author thanks the referee for careful reading of the manuscript and useful suggestions which allowed us to improve the present paper. This work is supported by the Russian Foundation for Basic Research, project no. 20-51-56015. 


\section{References}

[1] O.G. Avsyankin, $C^{*}$-algebra of integral operators with homogeneous kernels and oscillating coefficients. Mathematical Notes 99 (2016), no. 3, 345-353.

[2] O.G. Avsyankin, Volterra type integral operators with homogeneous kernels in weighted $L_{p}-$ spaces. Russian Mathematics 61 (2017), no. 11, 1-9.

[3] O.G. Avsyankin, Invertibility of multidimensional integral operators with bihomogeneous kernels. Mathematical Notes 108 (2020), no. 2, 277-281.

[4] O.G. Avsyankin, Compactness of some operators of convolution type in generalized Morrey spaces. Mathematical Notes 104 (2018), no. 3, 331-338.

[5] O.G. Avsyankin, N.K. Karapetyants, On the pseudospectra of multidimensional integral operators with homogeneous kernels of degree - n. Siberian Mathematical Journal 44 (2003), no. 6, 935-950.

[6] V.I. Burenkov, Recent progress in studying the boundedness of classical operators of real analysis in general Morrey-type spaces. I. Eurasian Mathematical Journal 3 (2012), no. 3, 11-32.

[7] V.I. Burenkov, Recent progress in studying the boundedness of classical operators of real analysis in general Morrey-type spaces. II. Eurasian Mathematical Journal 4 (2013), no. 1, 21-45.

[8] V.I. Burenkov, T.V. Tararykova, An analog of Young's inequality for convolutions of functions for general Morreytype spaces. Proc. Steklov Inst. Math. 293 (2016), 107-126.

[9] V.I. Burenkov, T.V. Tararykova, Young's inequality for convolutions in Morrey-type spaces. Eurasian Mathematical Journal 7 (2016), no. 2, 92-99.

[10] I.M. Gelfand, D.A. Raikov, G.E. Shilov, Commutative normed rings. Fizmatgiz, Moscow, 1960 (in Russian).

[11] A. Karapetyants, E. Liflyand, Defining Hausdorff operators on Euclidean spaces. Mathematical Methods in the Applied Sciences. Special Issue: Operator Theory and Harmonic Analysis 43 (2020), no. 16. 2020. P. 9487-9498.

[12] N. Karapetiants, S. Samko, Equations with involutive operators. Birkhäuser, Boston, Basel, Berlin, 2001.

[13] C.B. Morrey, On the solutions of quasi-linear elliptic partial differential equations. Trans. Amer. Math. Soc. 43 (1938), no. 1, 126-166.

[14] S.M. Umarkhadzhiev, Integral operators with homogeneous kernels in grand Lebesgue spaces. Mathematical Notes 102 (2017), no. 5, 710-721.

Oleg Gennadievich Avsyankin

Institute of Mathematics, Mechanics and Computer Sciences

Regional Mathematical Center

Southern Federal University

8a Mil'chakova St,

344090 Rostov-on-Don, Russia

E-mail: ogavsyankin@sfedu.ru

Received: 25.10.2019 